\title{
AFECTACIÓN AMBIENTAL POR LAS DESCARGAS DIRECTAS DE LAS AGUAS RESIDUALES SOBRE FUENTES HIDRICAS: CASO DE ESTUDIO TONA SANTANDER
}

\section{ENVIRONMENTAL IMPACT OF DIRECT WASTEWATER DISCHARGES ON WATER SOURCES: CASE STUDY TONA SANTANDER}

\author{
Nelson Andrey Navas Gallo ${ }^{(*)}$ y Constanza Silva Jaimes \\ Unidades Tecnológicas de Santander, Bucaramanga, Colombia. \\ ${ }^{(*)}$ e_mail: ingnavasg14@gmail.com
}

\begin{abstract}
RESUMEN
El objetivo del presente estudio fue evaluar la afectación al medio biótico, abiótico y demográfico causado por la generación y descarga de las aguas residuales domésticas sin un adecuado tratamiento sobre fuentes hídricas. Se realizaron análisis de los parámetros $\mathrm{DQO}, \mathrm{DBO}_{5}$, Sólidos Suspendidos Totales y Aceites y Grasas, en el vertimiento para conocer el cumplimiento de la normatividad Ambiental vigente Resolución 0631 de 2015, de igual forma se halló el índice de biodegradabilidad, donde se demostró que es de carácter poco biodegardable. Finalmente se realizó una identificación y evaluación de impactos ambientales de los componentes del ambiente respecto a las actividades cotidianas que se realizan en el municipio, y de esta forma se determinó la magnitud y afectación sobre los recursos ambientales del municipio de Tona Santander.
\end{abstract}

Palabras clave: agua residual, impacto ambiental, vertimiento.

\begin{abstract}
The objective of the present study was to evaluate the affectation of biotic, abiotic and demographic environment caused by the generation and discharge of domestic wastewater without adequate treatment on water sources. Analysis of the parameters COD, BOD, Total Suspended Solids, Dissolved Oxygen, Oils and Fats were carried out in the discharge. This was carried out to check compliance with the current Environmental regulations Resolution 0631 of 2015, in the same way the biodegradability index was investigated, where it was shown to be poorly biodegradable. Finally, an identification and evaluation of environmental impacts of the components of the environment with respect to the daily activities carried out in the municipality was carried out, and in this way the magnitude and impact on the environmental resources of the municipality of Tona Santander were determined.
\end{abstract}

Keywords: Wastewater, Environmental Impact, water source. 


\section{INTRODUCCIÓN}

Comenzando en la antigüedad el ser humano, ha venido realizando prácticas relativas a la generación de aguas residuales, desde sus inicios las diferentes sociedades desarrollaban sistemas de canales para recoger flujos de aguas y posteriormente, efectuaban diversas técnicas que la mejoraban de acuerdo a su necesidad. La primera instalación de saneamiento, fue el pozo ciego o pozo negro que apareció en Babilonia hacia 4000 a.C. la cual fue una simple excavación en el suelo donde depositaban los excrementos que se sistematizó a otras localidades del imperio y zonas rurales. (UNESCO, 2017).

El avance técnico y tecnológico que ha desarrollado el hombre, por décadas, se ha visto reflejado en el deterioro del ambiente, especialmente las fuentes hídricas, generando cambios en la calidad de vida, es así que en la década de los setenta, los sistemas socioeconómicos y políticos que lideraron prácticas impositivas a nivel mundial, iniciaron a preocuparse por la problemática que estaban generando a la sociedad, al sobreponer lo económico sobre el factor ambiental, de esta forma se fue implementando la importancia de estudiar las afectaciones que se generan al ambiente por las diferentes actividades industriales y residenciales. (Rodríguez et al., 2011).

Aun así, el recurso hídrico ha generado una solidaridad mundial a todos los niveles, sin distingo de raza, religión y cultura, pues con ello, se trata de preservar la vida como un derecho fundamental, apareciendo infinidad de grupos defensores del medio ambiente, que buscan generar conciencia en los diferentes actores sociales, políticos, catedráticos y comunales, surgiendo propuestas legislativas en pro de las metodologías de educación ambiental, desarrollo sostenible, tecnologías limpias, y buen aprovechamiento de los recursos naturales, que le permitieron a la sociedad, interactuar entre un proyecto y el medio ambiente a la hora de realizar cualquier actividad, interponiendo la normatividad vigente que rige cada territorio.

Todos los procesos y procedimientos de la plantas de tratamiento de aguas residuales, están ligados a la capacidad de cobertura que poseen las mismas en cada ente territorial, logrando eliminar gran parte de la carga contaminante que produce la comunidad de cada localidad, en Colombia existen Plantas de Tratamiento de Agua Residual, pero no son suficientes paras los más de mil cien (1100) municipios que posee, de igual forma las existentes, en un alto porcentaje no son eficientes y eficaces en su actividad principal, otro efecto es el aumento de población comparado con la cobertura limitada de las plantas existentes en el ámbito regional y nacional, las cuales realizan las descargas directas sobre fuentes hídricas, impactando directamente los recursos acuáticos como lo son: los ríos, lagos, quebradas y manantiales, los cuales desembocan en el mar u océano, e indirectamente impactando en la biota acuática y en la biodiversidad de los diferentes ecosistemas presentes. (Lizarazo y Orjuela, 2013).

En la actualidad el recurso hídrico demanda gran cantidad de consumo por el incremento de la población, pero en muchas oportunidades el agua presenta alteración en sus propiedades naturales por parte de la diversidad de actividades que ejecuta el hombre para su sustento, haciendo necesario la protección y preservación de este recurso, partiendo de este problema se busca generar conciencia para que se aumente el desarrollo de proyectos, donde se analice la afectación a las fuentes hídricas por las descargas de las aguas residuales sin tratamientos óptimos.

De igual forma la identificación y evaluación de impactos ambientales generados por el funcionamiento de diferentes actividades económicas que se realizan en los municipios es necesaria, estas actividades pueden alterar la calidad del agua directa o indirectamente, por lo cual se ve la necesidad que los entes gubernamentales desarrollen metodologías o proyectos, para conocer el grado de magnitud y afectación de cada uno de los factores ambientales los cuales comprenden elementos bióticos, abióticos y socioculturales que frecuentemente son alterados.

\section{METODOLOGÍA}

\section{Características de la zona de estudio.}

El estudio se desarrolló sobre la fuente hídrica Rio Tona, en el Departamento de Santander Colombia, el cual se encuentra localizado aproximadamente a 19 kilómetros de la ciudad de Bucaramanga capital del Departamento. La zona se encuentra ubicada sobre los 1100 metros a nivel del mar, con una temperatura comprendida entre los 13 y 19 grados centígrados, y una precipitación anual de 695 milímetros (Ramírez, 2016).

El monitoreo se ejecutó dando cumplimiento a los 
requisitos y protocolos contenidos en el instructivo para la toma de aguas residuales del Instituto de Hidrología, Meteorología y Estudios Ambientales; es necesario aclarar que para ejecutar los análisis referentes a la caracterización fisicoquímica, se realizó con un laboratorio acreditado, finalmente la revisión normativa se realizó conforme a lo establecido en la Resolución 631 de 2015 para cargas Correspondientes iguales o menores a $625.00 \mathrm{~kg} /$ día. (IDEAM, 2007).

Se realizaron muestras puntuales al vertimiento principal del municipio en diferentes tiempos, los rangos de tiempo fueron de (2) horas; durante un lapso de doce (12) horas en total, mezcladas proporcionalmente al caudal el cual se halló por el método Volumétrico. La captación de cada muestra tuvo un volumen de 500 mililitros, con la finalidad de elaborar una muestra compuesta, las cuales fueron dispuestas en un recipiente, con hielo a temperaturas entre un rango de 2 a 8 grados centígrados, garantizando la mínima contaminación o variación en sus características naturales.

Los parámetros ejecutados fueron $\mathrm{pH}$ (Standars methods $4500 \mathrm{H}+\mathrm{B}$ ), DQO (Reflejo cerradocolorimétrico Standars methods 5220 D), Oxígeno disuelto (Electrodo de membrana, standard methods $4500-\mathrm{O} \mathrm{G}), \mathrm{DBO}_{5}$ (Incubación a 5 días-electrodo de membrana, standard methods 5210 B, 4500 - O G), Solidos Suspendidos Totales (Gravimétrico - Secado A $103-105^{\circ} \mathrm{C}$, standard methods 2540 D), Grasas y Aceites (Extracción Soxhlet standard methods 5520 D).

Para elaborar la línea base ambiental se adquirió información primaria a través de visitas de campo en la zona perímetro de estudio, donde se analizó y se identificó los diferentes recursos, y las actividades cotidianas que se realizan en el municipio que generan alguna clase de impacto al ambiente. La información secundaria se basó en el Esquema de Ordenamiento Territorial, Plan de Desarrollo Regional.

La identificación de los impactos se realizó a través del método matricial, el cual establece la interacción en cada uno de los componentes (Aire, Agua, Suelo, Paisaje, Flora, Fauna, Económico y Cultural), los cuales son afectados por las actividades que se realizan en la zona de estudio, en cada celda donde se generó una interacción se trazó una equis (x) indicando de esta forma que allí se encontraba un impacto negativo o positivo.

La evaluación de impactos ambientales se realizó a través del método de Leopold. El cual es un método indirecto que califica las interacciones entre el proyecto y el ambiente. La matriz contiene dobles entradas en las columnas se ubicaron las actividades que se realizan en la zona de estudio, y en las filas los componentes ambientales, con la información de las interacciones que se generan entre los componentes ambientales y las actividades de la zona de estudio, se procedió a calificar cada interacción a través de tres criterios. (Arboleda, 2008):

Clase: Indica el tipo y signo del impacto positivo $(+)$ o negativo (-).

Magnitud: Representa al grado o nivel de alteración que se genera en el factor ambiental por la ejecución de la actividad, se asignó valores numéricos comprendidos entre 1 y 10 , donde 1 es la alteración mínima y 10 la alteración máxima.

Importancia: Analiza la relevancia que tiene factor ambiental en la zona de estudio, se asignó valores comprendidos entre 1 y 10 , donde 1 es insignificante y 10 es severo.

Ejecutada la calificación se procedió a realizar la síntesis de los impactos ambientales obtenidos. Para realizar el análisis de la evaluación se halló la media, desviación estándar y Rango de la media, con lo cual se identificaron: las actividades que mayor número de impactos generan, los factores ambientales que se impactan, el número de impactos negativos y positivos, y la calificación numérica de cada uno de los impactos.

\section{RESULTADOS Y DISCUSIÓN}

Análisis de los parámetros fisicoquímicos y sus valores limites máximo permisibles de las aguas residuales domésticas, de las aguas industriales, comerciales o de servicios.

En la Tabla 1 se observa los resultados de la caracterización del vertimiento principal del municipio de Tona, los cuales se compararon con los valores límites para cargas menores o iguales a $625.00 \mathrm{Kg} /$ díaDBO 5 según artículo 8 de la Resolución 0631 del año 2015. 
Tabla 1. Comparación análisis fisicoquímicos del vertimiento (Autoría propia.)

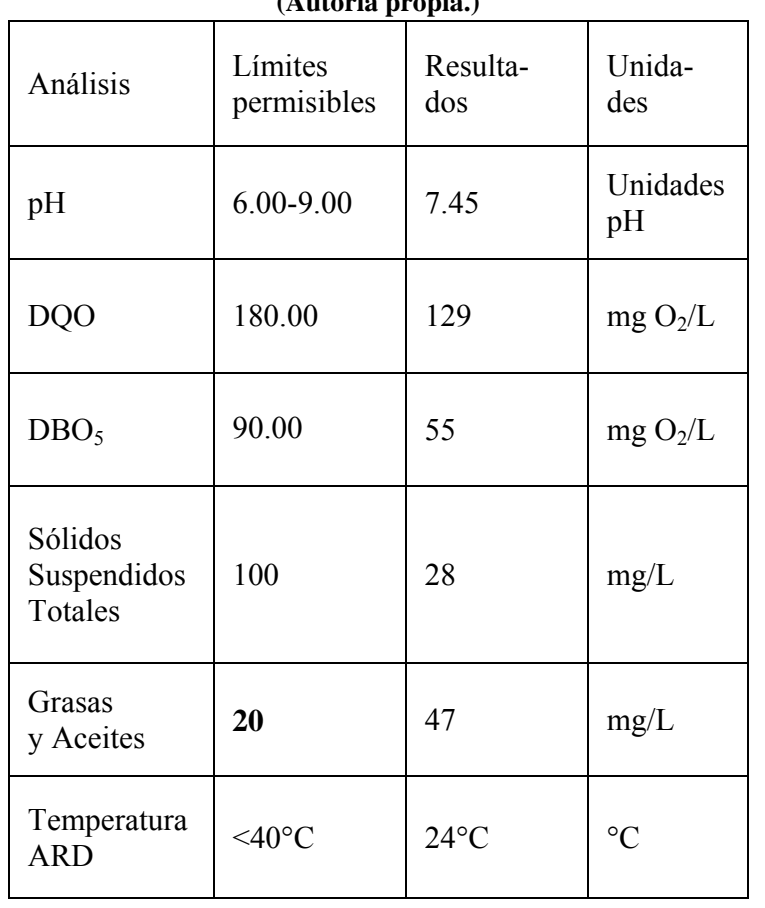

Se observa que el único parámetro que supera los límites máximos permisibles son las grasas y aceites las cuales según Romero (2009) se definen como "los compuestos de carbono, hidrogeno y oxigeno que flotan en el agua residual, que interfieren en la actividad biológica (...). Este aumento se puede presentar por la presencia de restaurantes, que aumentan su distribución de servicios por el número de turísticas que visitan al municipio, resaltando que el aumento de este parámetro proviene generalmente de mantequillas margarinas, aceites y carnes (Romero, 2009)

La Demanda Bioquímica de Oxigeno se refiere a la cantidad de oxigeno que requieren los microorganismos en el momento de proliferar en el agua residual y alimentarse de la materia orgánica, en condiciones aerobias. Mientras que la Demanda Química de Oxígeno se usa para medir el oxígeno equivalente a la materia orgánica oxidable, es decir mide toda la materia orgánica del agua, la biodegradable y la no biodegradable. (Rojas, 2008)

Al analizar los valores de los parámetros anteriormente mencionados, no superan los máximos permisibles, pero al calcular el índice de biodegradabilidad y comparar con la Tabla 2. La cual relaciona la $\mathrm{DBO}_{5} /$ DQO, se demostró que el resultado es 0.42 por lo cual se puede afirmar que es poco biodegradable
Tabla 2. Carácter de Biodegradabilidad del Agua Residual (Ardila et al., 2016)

\begin{tabular}{|l|l|}
\hline $\mathrm{DBO}_{5} / \mathrm{DQO}$ & Carácter \\
\hline$>0.8$ & Muy biodegradable \\
\hline $0.7-0.8$ & Biodegradable \\
\hline $0.3-0.7$ & Poco Biodegradable \\
\hline$<0.3$ & No Biodegradable \\
\hline
\end{tabular}

El oxígeno disuelto es uno de los parámetros fundamentales para conocer la calidad del agua, el valor de la muestra corresponde a $1.02 \mathrm{mg} / \mathrm{L}$, este valor se encuentra por debajo de los valores básicos de oxigeno establecidos para aguas normales naturales, las cuales, de acuerdo a Ardila et al., (2016), deben estar en un rango comprendido entre 7.0 y $8.0 \mathrm{mg} / \mathrm{L}$, lo cual indica una alteración o contaminación alta.

\section{Identificación y Evaluación de Impactos Ambientales.}

Se realizó una caracterización para conocer las actividades principales del municipio, la cuales contribuyen al aumento de las aguas residuales y a la afectación de la zona de la fuente hídrica. Las actividades identificadas son: Turismo, Fabricación de lácteos, cría y levante de pollos, producción piscícola, actividades de siembra y producción de cultivos de café, cacao, frijol, producción bovina y porcícola.

En la Tabla 3 se plasma las interacciones existentes entre las actividades y los factores componentes ambientales en la zona de estudio.

En la Tabla 4 se presentan los resultados del número de interacciones y síntesis de las actividades que se realizan en el municipio en donde se detalla que las interacciones positivas son 15 y 83 negativas, en la Ilustración 1 se observa que las actividades relacionadas con la siembra de cultivos, generación de aguas residuales, producción porcícola, bovina y piscícola son las actividades que mayor número de impactos negativos genera al ambiente. Con referencia a las interacciones positivas se halló 15 las cuales se encuentran uniformemente distribuidas en las diferentes actividades.

La síntesis de las actividades demuestra que todas son de tipo negativo, donde la desviación estándar corresponde a 114.53 , con un rango entre -129 y -506 , demostrando 
de esta forma que las actividades que más interfieren en la afectación del ambiente son: la generación de aguas residuales del municipio las cuales proceden de las diferentes viviendas, comercios e instituciones; de igual forma se demuestra que los diferentes cultivos que se llevan en la región como lo son café, cacao entre otros representan una amenaza para el ambiente por la aplicación de diferentes insecticidas, que indirectamente por medio de la escorrentía puede llegar a generar afectaciones al recurso hídrico y suelo.

Tabla 3. Identificación de impactos Ambientales (Autoría propia)

\begin{tabular}{|c|c|c|c|c|c|c|c|c|c|}
\hline \multicolumn{10}{|c|}{ propla } \\
\hline $\begin{array}{l}\text { Factor } \\
\text { Ambient } \\
\text { al }\end{array}$ & Actividades & 1 & 2 & 3 & 4 & 5 & 6 & 7 & 8 \\
\hline \multirow{4}{*}{ Agua } & $\begin{array}{l}\text { Disminución } \\
\text { recurso hídrico }\end{array}$ & $\mathrm{x}$ & & $\mathrm{x}$ & & $\mathrm{x}$ & $\mathrm{x}$ & & $\mathrm{x}$ \\
\hline & $\begin{array}{l}\text { Contaminación del } \\
\text { agua }\end{array}$ & $\mathrm{x}$ & $\mathrm{x}$ & $\mathrm{x}$ & $\mathrm{x}$ & $\mathrm{x}$ & $\mathrm{x}$ & $\mathrm{x}$ & $\mathrm{x}$ \\
\hline & $\begin{array}{l}\text { Alteración } \\
\text { parámetros } \\
\text { fisicoquímicos del } \\
\text { agua }\end{array}$ & $\mathrm{x}$ & & $\mathrm{x}$ & & & $\mathrm{x}$ & & $\mathrm{x}$ \\
\hline & $\begin{array}{l}\text { Cambio uso del } \\
\text { agua }\end{array}$ & & $\mathrm{x}$ & & $\mathrm{x}$ & $\mathrm{x}$ & $\mathrm{x}$ & $\mathrm{x}$ & $\mathrm{x}$ \\
\hline \multirow{3}{*}{ Aire } & $\begin{array}{l}\text { Deterioro de la } \\
\text { Calidad del Aire }\end{array}$ & $\mathrm{x}$ & $\mathrm{x}$ & & $\mathrm{x}$ & $\mathrm{x}$ & $\mathrm{x}$ & $\mathrm{x}$ & $\mathrm{x}$ \\
\hline & $\begin{array}{l}\text { Generación de } \\
\text { Olores Ofensivos }\end{array}$ & $\mathrm{x}$ & & $\mathrm{x}$ & $\mathrm{x}$ & $\mathrm{x}$ & $\mathrm{x}$ & & $\mathrm{x}$ \\
\hline & $\begin{array}{l}\text { Generación de } \\
\text { ruido }\end{array}$ & & & $\mathrm{x}$ & & & $\mathrm{x}$ & & \\
\hline \multirow{3}{*}{ Suelo } & $\begin{array}{l}\text { Contaminación del } \\
\text { suelo }\end{array}$ & $\mathrm{x}$ & & $\mathrm{x}$ & $\mathrm{x}$ & & & & $\mathrm{x}$ \\
\hline & Erosión & & & & & $\mathrm{x}$ & $\mathrm{x}$ & $\mathrm{x}$ & $\mathrm{x}$ \\
\hline & $\begin{array}{l}\text { Alteración } \\
\text { fisicoquímica del } \\
\text { suelo }\end{array}$ & & & & $\mathrm{x}$ & $\mathrm{x}$ & $\mathrm{x}$ & $\mathrm{x}$ & $\mathrm{x}$ \\
\hline \multirow{2}{*}{ Paisaje } & $\begin{array}{l}\text { Alteración del } \\
\text { paisaje }\end{array}$ & $\mathrm{x}$ & & & $\mathrm{x}$ & $\mathrm{x}$ & $\mathrm{x}$ & $\mathrm{x}$ & $\mathrm{x}$ \\
\hline & $\begin{array}{l}\text { Modificación de } \\
\text { hábitats }\end{array}$ & $\mathrm{x}$ & & & & $\mathrm{x}$ & $\mathrm{x}$ & $\mathrm{x}$ & $\mathrm{x}$ \\
\hline \multirow{2}{*}{ Fauna } & $\begin{array}{ll}\begin{array}{l}\text { Migración } \\
\text { especies }\end{array} & \text { de } \\
\end{array}$ & $\mathrm{x}$ & $\mathrm{x}$ & & & $\mathrm{x}$ & $\mathrm{x}$ & $\mathrm{x}$ & $\mathrm{x}$ \\
\hline & $\begin{array}{l}\text { Fragmentación de } \\
\text { hábitats }\end{array}$ & $\mathrm{x}$ & $\mathrm{x}$ & & $\mathrm{x}$ & $\mathrm{x}$ & $\mathrm{x}$ & $\mathrm{x}$ & $\mathrm{x}$ \\
\hline \multirow[b]{2}{*}{ Flora } & $\begin{array}{l}\text { Disminución de la } \\
\text { cobertura vegetal }\end{array}$ & $\mathrm{x}$ & $\mathrm{x}$ & & $\mathrm{x}$ & $\mathrm{x}$ & $\mathrm{x}$ & $\mathrm{x}$ & $\mathrm{x}$ \\
\hline & $\begin{array}{l}\text { Extinción de } \\
\text { especies vegetales } \\
\text { endémicas }\end{array}$ & $\mathrm{x}$ & & & & & & & \\
\hline \multirow{3}{*}{ Social } & $\begin{array}{l}\text { Generación de } \\
\text { empleo }\end{array}$ & $\mathrm{x}$ & $\mathrm{x}$ & $\mathrm{x}$ & $\mathrm{x}$ & $\mathrm{x}$ & $\mathrm{x}$ & $\mathrm{x}$ & $\mathrm{x}$ \\
\hline & $\begin{array}{ll}\text { Incremento } & \text { de } \\
\text { ingresos en la } \\
\text { población local }\end{array}$ & & $\mathrm{x}$ & $\mathrm{x}$ & $\mathrm{x}$ & $\mathrm{x}$ & $\mathrm{x}$ & $\mathrm{x}$ & $\mathrm{x}$ \\
\hline & Adaptación cultural & & $\mathrm{x}$ & & & & & & \\
\hline
\end{tabular}

Referencias: 1. Generación de aguas Residuales; 2. Actividades de Turismo; 3. Fabricación de lácteos; 4. Cría y levante de pollos (avicultura); 5. Producción porcícola (porcicultura); 6. Siembra y producción de café, cacao, frijol; 7. Producción bovina (Ganadería); 8. Producción piscícola (piscicultura)
Tabla 4. Síntesis Actividades Zona de estudio (Autoría propia)

\begin{tabular}{|l|l|l|l|l|}
\hline $\begin{array}{l}\text { Actividades } \\
\text { identificadas en } \\
\text { la zona objeto } \\
\text { de estudio }\end{array}$ & $\begin{array}{l}\text { Interac- } \\
\text { cionesn } \\
\text { egati- } \\
\text { vas (-) }\end{array}$ & $\begin{array}{l}\text { Interac- } \\
\text { cionesp } \\
\text { ositi- } \\
\text { vas (+) }\end{array}$ & $\begin{array}{l}\text { Síntesis } \\
\text { negati- } \\
\text { vas (-) }\end{array}$ & $\begin{array}{l}\text { Síntesis } \\
\text { positi- } \\
\text { vas (+) }\end{array}$ \\
\hline $\begin{array}{l}\text { Generación de } \\
\text { aguas } \\
\text { residuales }\end{array}$ & 12 & 1 & 506 & 0 \\
\hline $\begin{array}{l}\text { Actividades de } \\
\text { Siembra y y } \\
\text { producción de } \\
\text { café, cacao, } \\
\text { frijol }\end{array}$ & 14 & 2 & 401 & 0 \\
\hline $\begin{array}{l}\text { Producción } \\
\text { porcícola }\end{array}$ & 12 & 2 & 337 & 0 \\
\hline $\begin{array}{l}\text { Producción } \\
\text { piscícola }\end{array}$ & 14 & 2 & 300 & 0 \\
\hline $\begin{array}{l}\text { Producción } \\
\text { bovina }\end{array}$ & 10 & 2 & 239 & 0 \\
\hline $\begin{array}{l}\text { Cría y levante } \\
\text { de pollos }\end{array}$ & 8 & 2 & 231 & 0 \\
\hline Turismo & 7 & 2 & 182 & 0 \\
\hline $\begin{array}{l}\text { Fabricación de } \\
\text { lácteos }\end{array}$ & 6 & 2 & 129 & 0 \\
\hline TOTAL & 83 & 15 & 2325 & 0 \\
\hline
\end{tabular}

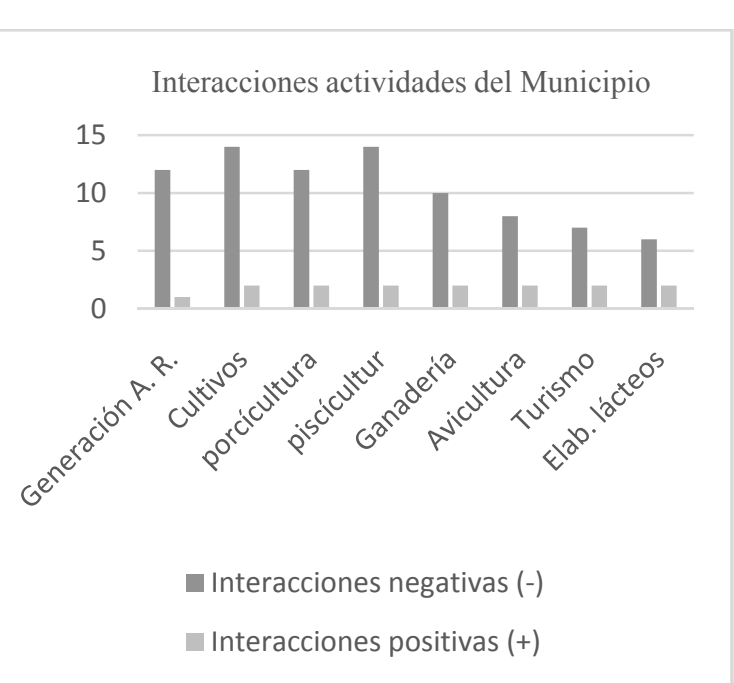

Ilustración 1. Interacciones por Actividad (Elaboración propia).

En la Tabla 5 se plasma la afectación por componentes ambientales donde se determinó que el componente que presenta mayor número de interacciones es el recurso agua con un total de 23 interacciones negativas, seguido del recurso aire y suelo. Se evidenció que el factor económico presentó 15 interacciones, lo que representa 15 impactos de clase positiva.

Se determinó que el factor ambiental que mayor número de impactos negativos presenta es el agua, 
precedido del recurso suelo y fauna, por lo cual se hace necesario intervenir a través de la aplicación de fichas de manejo de protección, donde plasmen programas para mitigar y/o prevenir los impactos. Es importante detallar que el factor económico es el único que representa impactos positivos por el incremento de ingresos en la población del municipio.

Tabla 5. Síntesis Factores Ambientales (Elaboración propia).

\begin{tabular}{|l|l|l|l|l|}
\hline $\begin{array}{l}\text { Componente } \\
\text { ambiental }\end{array}$ & $\begin{array}{l}\text { Interac- } \\
\text { ciones } \\
\text { negativas } \\
(-)\end{array}$ & $\begin{array}{l}\text { Interac- } \\
\text { ciones } \\
\text { positivas } \\
(+)\end{array}$ & $\begin{array}{l}\text { Síntesis } \\
\text { negativas } \\
(-)\end{array}$ & $\begin{array}{l}\text { Síntesis } \\
\text { positivas } \\
(+)\end{array}$ \\
\hline Agua & 23 & 0 & 861 & 0 \\
\hline Suelo & 13 & 0 & 488 & 0 \\
\hline Fauna & 13 & 0 & 408 & 0 \\
\hline Aire & 15 & 0 & 324 & 0 \\
\hline Paisaje & 11 & 0 & 276 & 0 \\
\hline Flora & 7 & 0 & 208 & 0 \\
\hline Cultural & 1 & 0 & 12 & 0 \\
\hline Económico & 0 & 15 & 0 & 252 \\
\hline$\sum$ & 83 & 15 & 2577 & 252 \\
\hline
\end{tabular}

\section{CONCLUSIONES}

El índice de biodegradabilidad del vertimiento analizado, se calificó como poco biodegradable, lo cual demuestra que se deben realizar tratamientos eficientes antes de ser vertidos sobre la fuente hídrica, para evitar alteración tanto en el recurso hídrico como en la biota acuática, suelo, aire, al igual que en la calidad de vida de los habitantes del municipio.

El total de parámetros fisicoquímicos no se encuentran por debajo de los límites máximos permisibles reglamentados en la Resolución 0631 del año 2015, por lo cual es necesario que las autoridades ambientales realicen un seguimiento semestral de acuerdo a la normatividad vigente, para implementar medidas que contribuyan al cumplimiento de la Resolución en materia de vertimientos.

A través de la evaluación de impactos ambientales realizada respecto con las actividades cotidianas que se realizan en el municipio, se concluyó que los recursos agua, aire, suelo y fauna son los más afectados.

Los resultados obtenidos del presente estudio muestran la importancia para que las autoridades municipales y ambientales competentes desarrollen estudios ambientales que evalúen el estado de actual de los recursos ambientales en el municipio, y de esta forma implementen medidas para minimizar, corregir y/o compensar los impactos generados sobre el medio.

\section{REFERENCIAS}

Arboleda, J. (2008). Manual para la evaluación de impacto ambiental de proyectos, obras o actividades EIA. Medellin. Obtenido de https://bit.ly/2OZikJt

Ardila, A. N., Arriola, E., Reyes, J., Berrio, E., y Fuentes, G. (2016). Mineralización De Etilenglicol Por Foto-Fenton Asistido Con Ferrioxalato. Rev. Int. Contam. Ambie, 32(2), 213-226.

IDEAM. (2007). Toma de muestras de aguas residuales. Bogotá D.C: Instituto de Hidrología, Meteorología y Estudios Ambientales. Obtenido de https://bit.ly/3g6KCh7

Lizarazo, J. y Orjuela, M. (2013). Sistemas de plantas de tratamiento de aguas residuales en Colombia. Bogotá D.C: Universidad Nacional de Colombia. Obtenido de https://bit.ly/2BDKU06

Ramírez Aldana, C. L. (2016). Todas y todos construimos futuro: Plan de Desarrollo 20162019. Tona: Alcaldía Tona. Obtenido de https://bit.ly/3jKgc6E

Rodríguez, A., Martínez, M., Martínez, I., Fundora, H., y Guzmán, T. (2011). Desarrollo tecnológico, impacto sobre el medio ambiente y la salud.Revista Cubana de Higiene y Epidemiología, 49(2):308-319.

Romero, J. A. (2009). Tratamiento de las Aguas Residuales. Bogotá: Lemoine Editores.

UNESCO. (2017). Aguas residuales el recurso desaprovechado. París: Organización de las Naciones Unidas para la Educación, la Ciencia y la Cultura. Obtenido de https://unesdoc.unesco.org/ark:/48223/pf00002476 47 
Tipo de Publicación: NOTA TÉCNICA.

Trabajo recibido el 09/09/2020 y aprobado para su publicación el 15/01/2021.

\section{COMO CITAR}

Navas Gallo, N. A. y Constanza Silva Jaimes, C. (2021). Afectación ambiental por las descargas directas de las aguas residuales sobre fuentes hídricas: Caso de estudio Tona
SantanderCuadernos del CURIHAM,27,85-91.

DOI: https://doi.org/10.35305/curiham.v27i.163

Esta es unanota técnica de acceso abierto bajo licencia: Creative Commons Atribución - No Comercial - Compartir Igual 4.0 Internacional (CC $B Y-N C-S A$

4.0)

(https://creativecommons.org/licenses/by-ncsa/4.0/deed.es) 